\title{
SPHERICAL CURVES AND QUADRATIC RELATIONSHIPS FOR SPECIAL FUNCTIONS
}

\author{
EVEN MEHLUM ${ }^{1}$ and JET WIMP ${ }^{2}$
}

(Received 27 October 1983; revised 8 May 1984)

\begin{abstract}
We show that the position vector of any 3-space curve lying on a sphere satisfies a third-order linear (vector) differential equation whose coefficients involve a single arbitrary function $A(s)$. By making various identifications of $A(s)$, we are led to nonlinear identities for a number of higher transcendental functions: Bessel functions, Horn functions, generalized hypergeometric functions, etc. These can be considered natural geometrical generalizations of $\sin ^{2} t+\cos ^{2} t=1$. We conclude with some applications to the theory of splines.
\end{abstract}

\section{Introduction}

Traditionally, identities involving special functions have been demonstrated using analytical methods, such as power series arguments and manipulations with integrals and differential equations. In fact Watson's classic treatise on Bessel functions [12] is a virtual compendium of such techniques. In the past 25 years or so, algebraic approaches, particularly those based on the theory of group representations, have become popular, and a wealth of previously hidden results have been revealed this way. Prominent names in these efforts are those of Weisner, Koornwinder, Miller, and Dunkl. (The book [2] contains many references to such results.) It has been subsequently discovered that many results obtained by algebraic methods may also be proved using analytical techniques, although, as

\footnotetext{
${ }^{1}$ Central Institute for Industrial Research, Oslo, Norway.

${ }^{2}$ Department of Mathematical Sciences, Drexel University, Philadelphia, Pennsylvania 19104, U.S.A.

(c) Copyright Australian Mathematical Society 1985, Serial-fee code 0334-2700/85
} 
may be expected, the analytical derivations are very tricky. Askey's analytical proof [1] of a result of Koornwinder on Jacobi polynomials is a good example of such an undertaking.

What seems to have been relatively overlooked is the possibility of using geometric arguments to derive identities for special functions. While it is true that algebraic methods utilize certain geometric concepts, such as symmetry groups on spheres, the reasoning in the algebraic approach is not geometrical.

In this paper we examine a way of using purely geometrical considerations, namely, properties of spherical curves, to derive special-function identities.

In the reference [10] it was shown how a certain problem in the theory of nonlinear splines led to a third order differential equation for the position vector of a curve which lay on a sphere. When the differential equation was solved and the condition that the sum of the squares of the components of the solution equals a constant was imposed, a nonlinear relation involving confluent hypergeometric functions of two variables was obtained. This analysis, whose physical implications are discussed in Section 6 of this paper, has turned out to be enormously fruitful and leads to a wide variety of relations, as we shall see.

The results may be considered to be geometric generalizations of

$$
\sin ^{2} t+\cos ^{2} t=1,
$$

but they are very exotic, and we suspect that attempts to demonstrate them by analytical techniques will encounter great obstacles. Our identities, particularly those involving the confluent functions $\Phi_{1}$ and $\Phi_{2}$, seem to have no parallel in the classical literature.

After presenting our approach and a number of examples, we close with an application to nonlinear splines and suggestions for future research.

We use the notation $w$ for vectors in 3-space, and $w^{(t)}$ denotes the $i$ th component of $\mathbf{w}$.

Our notation for special functions is exclusively that of the Bateman manuscript volumes, [5].

\section{Spherical curves}

Let $\rho$ be the position vector of a curve $(\gamma)$ in 3-space, parameterized by arc length $s$. We assume $\rho$ is suitably smooth. $(t, n, b)$ will denote as usual, the moving trihedral of the curve and $\kappa, \tau$ the curvature and torsion respectively. It is known (see, for instance [11]) that $\rho$ satisfies a fourth order differential equation (in $s$ ) with coefficients involving $\kappa$ and $\tau$. However, as we shall see, a simpler equation is possible when $(\gamma)$ lies on a sphere centered at $\mathbf{0}$. Since $(\mathbf{t}, \mathbf{n}, \mathbf{b})$ is an orthonormal 
system, we may write

$$
(d / d s)^{n} \rho=a_{n} \mathbf{t}+b_{n} \mathbf{n}+c_{n} \mathbf{b},
$$

for some functions $a_{n}, b_{n}, c_{n}$. Differentiating, using the Frenet-Serret formulas, [11], and equating coefficients of $(t, n, b)$ results in the equations

$$
\begin{aligned}
& a_{n+1}=a_{n}^{\prime}-\kappa b_{n}, \\
& b_{n+1}=a_{n} \kappa+b_{n}^{\prime}-\tau c_{n}, \\
& c_{n+1}=\tau b_{n}+c_{n}^{\prime} .
\end{aligned}
$$

When $n=1, a_{1}=1, b_{1}=c_{1}=0$, since $\rho^{\prime}=\mathbf{t}$. Putting $n=0$ in (2.2) and using these values gives

$$
\begin{aligned}
& 1=a_{0}^{\prime}-\kappa b_{0}, \\
& 0=a_{0} \kappa+b_{0}^{\prime}-\tau c_{0}, \\
& 0=\tau b_{0}+c_{0}^{\prime} .
\end{aligned}
$$

The condition that $(\gamma)$ lie on a sphere centered at the origin is

$$
\rho \cdot \rho^{\prime}=0, \text { i.e., } \rho \cdot \mathbf{t}=0 .
$$

Since $\rho^{\prime}=\mathbf{t}$ and $\rho^{\prime \prime}=\kappa \mathbf{n}$,

$$
\rho^{\prime} \cdot \boldsymbol{\rho}^{\prime}=1, \quad \rho^{\prime \prime} \cdot \rho^{\prime \prime}=\kappa^{2}, \quad \rho^{\prime} \cdot \rho^{\prime \prime}=0 .
$$

Furthermore, differentiating (2.4) gives

$$
\rho \cdot \rho^{\prime \prime}=-1 .
$$

The five conditions (2.4), (2.5), (2.6) will subsequently be used to derive the appropriate initial data for the problem.

Using (2.1) with $n=0$ and employing (2.4) shows $a_{0}=0$. Thus (2.3) reduces to

$$
\begin{aligned}
& 1=-\kappa b_{0}, \\
& 0=b_{0}^{\prime}-\tau c_{0}, \\
& 0=\tau b_{0}+c_{0}^{\prime} .
\end{aligned}
$$

The first two equations yield

$$
b_{0}=-1 / \kappa, \quad c_{0}=\kappa^{\prime} / \tau \kappa^{2}
$$

and the third requires

$$
\frac{\tau}{\kappa}-\left(\frac{\kappa^{\prime}}{\tau \kappa^{2}}\right)^{\prime}=0
$$

which is the known intrinsic equation for $\rho$ (see [3]). It is a necessary and sufficient condition for $(\gamma)$ to lie on a sphere. Expressing $\mathbf{t}, \mathbf{n}, \mathbf{b}$ in terms of $\boldsymbol{\rho}^{\prime}, \boldsymbol{\rho}^{\prime \prime}$, $\rho^{\prime \prime \prime}, \kappa$ and $\tau$ via the Serret-Frenet formulae and substituting the results, and (2.7), 
into (2.1), we obtain the differential equation

$$
\kappa^{\prime} \rho " \prime-\left(\tau^{2} \kappa+\kappa^{\prime 2} / \kappa\right) \rho^{\prime \prime}+\kappa^{\prime} \kappa^{2} \rho^{\prime}-\tau^{2} \kappa^{3} p=0,
$$

with $\kappa$ and $\tau$ related by (2.8).

The intrinsic equation (2.8) may actually be integrated once, as follows. Taking the dot product of (2.9) with $\rho$ and using (2.5) and (2.6) yields the equation

$$
\frac{1}{\kappa^{2}}+\frac{\kappa^{\prime 2}}{\kappa^{4} \tau^{2}}=R^{2}
$$

where $R$ is the radius of the sphere.

Now let

$$
\kappa=\alpha \sqrt{1+A^{2}}, \quad \alpha=1 / R,
$$

where $A$ is any positive differentiable function of $s$. Using (2.10) then shows that

$$
\tau= \pm A^{\prime} /\left(1+A^{2}\right)
$$

and putting (2.11), (2.12) in (2.9) gives our final equation

$$
\rho^{\prime \prime \prime}-\left(A^{\prime} / A\right) \rho^{\prime \prime}+\alpha^{2}\left(1+A^{2}\right) \rho^{\prime}-\left(\alpha^{2} A^{\prime} / A\right) \rho=0, \quad \alpha=1 / R .
$$

Initial vectors satisfying (2.4), (2.5), (2.6) are

$$
\rho(0)=(0,0,-1 / \alpha), \quad \rho^{\prime}(0)=(0,1,0), \quad \rho^{\prime \prime}(0)=(\alpha A(0), 0, \alpha) .
$$

It turns out that no generality is gained in our final formulas by choosing initial vectors more general than (2.14). The rest of this paper is devoted to an analysis of the system (2.13)-(2.14).

Note that when $A$ equals a constant, the torsion vanishes and $\rho$ lies in a plane. The relation $\rho^{(1) 2}+\rho^{(2) 2}=R^{2}$ is then, essentially, (1.1).

\section{Some ${ }_{1} F_{2}$ formulas}

We take $A(s)=a e^{b s}$ and make a change of variable and parameter,

$$
e^{2 b s}=t, \quad p(s)=\mathbf{h}(t), \quad \nu=\alpha / 2 b,
$$

to get

$$
2 t^{3} \mathbf{h}^{\prime \prime \prime}+5 t^{2} \mathbf{h}^{\prime \prime}+\left[\left(1+2 \nu^{2}\right) t+2 \nu^{2} a^{2} t^{2}\right] \mathbf{h}^{\prime}-\nu^{2} \mathbf{h}=\mathbf{0} .
$$

We consider the corresponding scalar equation

$$
2 t^{3} h^{\prime \prime \prime}+5 t^{2} h^{\prime \prime}+\left[\left(1+2 \nu^{2}\right) t+2 \nu^{2} a^{2} t^{2}\right] h^{\prime}-\nu^{2} h=0 .
$$

The method of Frobenius is applicable and shows this equation has a fundamental set $\left(h_{1}, h_{2}, h_{3}\right)$ of the form

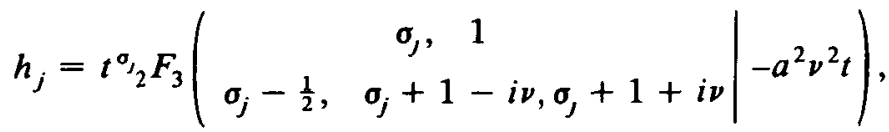

$$
\begin{aligned}
& \sigma_{1}=\frac{1}{2}, \sigma_{2}=i \nu, \sigma_{3}=-i \nu .
\end{aligned}
$$

(Note that these ${ }_{2} F_{3}$ 's are actually ${ }_{1} F_{2}$ 's.) 
The Wronskian of these solutions is found to be

$$
W(t)=-2 i \nu\left(\nu^{2}+\frac{1}{4}\right) t^{-5 / 2} .
$$

(this follows by using Abel's formula [7, page 119] and estimating the determinant by its leading powers of $t$ ).

The conditions (2.14) at $s=0$ become conditions at $t=1$,

$$
\mathbf{h}(1)=\left(0,0,-\frac{1}{2 b \nu}\right), \quad \mathbf{h}^{\prime}(1)=\left(0, \frac{1}{2 b}, 0\right), \quad \mathbf{h}^{\prime \prime}(1)=\left(\frac{a \nu}{2 b},-\frac{1}{2 b}, \frac{\nu}{2 b}\right)
$$

and $\mathbf{h}(t)$ may be conveniently represented by means of the vector determinant

$$
\mathbf{h}(t)=\frac{-1}{2 b W(1)}\left|\begin{array}{llll}
(0,0,0) & h_{1}(t) & h_{2}(t) & h_{3}(t) \\
(0,0,-1 / \nu) & h_{1}(1) & h_{2}(1) & h_{3}(1) \\
(0,1,0) & h_{1}^{\prime}(1) & h_{2}^{\prime}(1) & h_{3}^{\prime}(1) \\
(a \nu,-1, \nu) & h_{1}^{\prime \prime}(1) & h_{2}^{\prime \prime}(1) & h_{3}^{\prime \prime}(1)
\end{array}\right| .
$$

We shall not pursue the evaluation of the general determinant (3.5) here, but shall look at what happens when $a \rightarrow 0$. Replace $t$ by $t / a^{2}$. The derivatives of $h_{j}$ are easy to estimate and we have

$$
\begin{aligned}
\mathbf{h}\left(t / a^{2}\right):= & \mathbf{g}(t)=\left|\begin{array}{llll}
(0,0,0) & g_{1}(t) & g_{2}(t) & g_{3}(t) \\
(0,0,-1 / \nu) & 1 & 1 & 1 \\
(0,1,0) & \frac{1}{2} & i \nu & -i \nu \\
(a \nu,-1, \nu) & -\frac{1}{4} & i \nu(i \nu-1) & i \nu(i \nu+1)
\end{array}\right| \\
& \cdot \frac{\left(1+O\left(a^{2}\right)\right)}{4 i b \nu\left(\nu^{2}+\frac{1}{4}\right)},
\end{aligned}
$$

where $g_{j}(t):=h_{j}\left(t / a^{2}\right)$. Since $\nu>0, g_{2}=\bar{g}_{3}$. Adding $\nu^{2}$ times the second row, and the third, to the fourth produces an orthonormal set of vectors in the first column. Expanding by minors of this column gives a result that we write as

$$
\begin{aligned}
\mathbf{g}=\frac{1+O\left(a^{2}\right)}{2 b \nu}\{- & (0,0,1) \operatorname{Re} g_{2}+(0,1,0) \operatorname{Im} g_{2} \\
& \left.+\frac{\left(\nu^{2} a, 0,0\right)}{\left(\nu^{2}+1 / 4\right)}\left[g_{1}-\operatorname{Re} g_{2}-\frac{1}{2 \nu} \operatorname{Im} g_{2}\right]\right\} .
\end{aligned}
$$

Now $\mathbf{g} \cdot \mathbf{g}=R^{2}=\alpha^{-2}=\left(4 \nu^{2} b^{2}\right)^{-1}$. This condition, that the curve lies on a sphere, implies an exotic relation between certain ${ }_{1} F_{2}$ 's. To find it, we let $a \rightarrow 0$, replace $t$ by $t / \nu^{2}$, and take the dot product. The result is the identity $(t \geqslant 0)$

$$
\left|{ }_{1} F_{2}\left(\begin{array}{c}
i \nu \\
\frac{1}{2}+i \nu, 1+2 i \nu
\end{array} \mid-t\right)\right|^{2}+\frac{\nu^{2} t}{\left(\nu^{2}+\frac{1}{4}\right)^{2}}\left[{ }_{1} F_{2}\left(\begin{array}{c}
\frac{1}{2} \\
\frac{3}{2}+i \nu, \frac{3}{2}-i \nu
\end{array} \mid-t\right)\right]^{2}=1 .
$$


Another interesting formula involving ${ }_{1} F_{2}$ 's results on choosing

$$
A(s)=(s+\varepsilon)^{-1} \text {. }
$$

Making the change of variable

$$
s+\varepsilon=t, \quad \rho(s)=\mathbf{h}(t)
$$

gives a differential equation whose scalar analogue is

$$
t h^{\prime \prime \prime}+h^{\prime \prime}+\alpha^{2}(t+1 / t) h^{\prime}+\alpha^{2} h=0 .
$$

This has a basis of solutions

$$
\begin{aligned}
& h_{\jmath}=t_{\sigma_{2}} F_{3}\left(\begin{array}{c}
1,\left(\sigma_{j}+1\right) / 2 \\
\left(\sigma_{j}+2\right) / 2,\left(\sigma_{j}+1+i \alpha\right) / 2,\left(\sigma_{j}+1-i \alpha\right) / 2
\end{array} \mid-\alpha^{2} t^{2} / 4\right) \text {, } \\
& \sigma_{1}=0, \sigma_{2}=1+i \alpha, \sigma_{3}=1-i \alpha,
\end{aligned}
$$

and $s=0$ corresponds to $t=\varepsilon$ and $\rho^{\prime \prime}(0)=(\alpha / \varepsilon, 0, \alpha)$. Estimating $h_{j}(\varepsilon)$ is easy. Also $W(t)=-2 i \alpha\left(1+\alpha^{2}\right) / t$. We find, as before, that $\mathbf{h}$ may be represented by the vector determinant

$$
\mathbf{h}=\frac{(1+o(\varepsilon))}{2 i \alpha\left(1+\alpha^{2}\right)}\left|\begin{array}{llll}
(0,0,0) & h_{1} & h_{2} & h_{3} \\
(0,0,-1 / \alpha) & 1 & 0 & 0 \\
(0,1,0) & 0 & (1+\alpha) \varepsilon^{i \alpha} & (1-i \alpha) \varepsilon^{-i \alpha} \\
(\alpha, 0,0) & 0 & i \alpha(1+i \alpha) \varepsilon^{i \alpha} & -i \alpha(1-i \alpha) \varepsilon^{-i \alpha}
\end{array}\right|
$$

Expanding the determinant, taking its dot product with itself and letting $\varepsilon \rightarrow 0$ gives

$$
\mathbf{h} \cdot \mathbf{h}=\frac{1}{\alpha^{2}}=\frac{h_{1}^{2}}{\alpha^{2}}+\frac{1}{\left(1+\alpha^{2}\right)}\left|h_{2}\right|^{2} .
$$

Through straightforward series manipulations using the formulas in [5, volume 2, page 11] we may express the ${ }_{1} F_{2}$ 's in terms of Bessel functions. Letting $t \rightarrow 2 t / \alpha, \nu=\alpha / 2$ gives an unexpected result which, however, is a "natural" geometric generalization of

$$
\sin ^{2} t+\cos ^{2} t=1
$$

It is

$$
\begin{gathered}
\left|\frac{d}{d t}\left[t^{1-2 i \nu} J_{-1 / 2+i \nu}(t) J_{1 / 2-i \nu}(t)\right]\right|^{2}+4 t^{2}\left|J_{1 / 2+i \nu}(t) J_{-1 / 2+i \nu}(t)\right|^{2} \\
=\frac{4 \cosh ^{2} \nu \pi}{\pi^{2}}, \quad \nu \geqslant 0, t \geqslant 0,
\end{gathered}
$$

and the former equation results on putting $\nu=0$.

As a referee has pointed out, this formula may also be demonstrated by using the known differential properties (Wronskian relations) for Bessel functions. 
Whether the other formulas obtained in this paper also imply or express certain differential properties of the functions involved is not known. It seems doubtful, however, that this could be the case since these functions do not satisfy second order differential equations; nor are they, generally, expressible as products of functions that do, so the Wronskians involved must be expected to involve products of three, rather than two, functions.

\section{Horn functions}

The next choice is $A(s)=(s+\varepsilon)^{-1 / 2}$. Making again the substitution (3.7) gives an equation whose scalar counterpart is

$$
t h^{\prime \prime}+\frac{1}{2} h^{\prime \prime}+\alpha^{2}(1+t) h^{\prime}+\frac{1}{2} \alpha^{2} h=0 .
$$

The method of Frobenius reveals that this equation has a basis of solutions near $t=0$ with the behaviour,

$$
h_{1}=1+a_{1} t+\cdots, h_{2}=t+b_{1} t^{2}+\cdots, h_{3}=t^{3 / 2}+c_{1} t^{5 / 2}+\cdots .
$$

(It seems not to be feasible to find the coefficients in these expansions directly. We shall return later to the identification of $h_{j}$.) We form the vector determinant exactly as before, estimating things to order $\varepsilon^{1 / 2}$, take dot products and let $\varepsilon \rightarrow 0$. We find

$$
\left(h_{1}-a_{1} h_{2}\right)^{2}+\alpha^{2} h_{2}^{2}+\frac{16}{9} \alpha^{4} h_{3}^{2}=1 .
$$

We now return to the $h_{j}$. The equation may be solved by Laplace integrals of the form

$$
h(t)=\int_{\Gamma} e^{t u} \phi(u) d u,
$$

where $\Gamma$ is a path in the complex $u$-plane. Substituting (4.4) in (4.1) and performing a single integration by parts on the terms containing $t$ (this, by the way, requires that $e^{i u} u\left(u^{2}+\alpha^{2}\right) \phi$ vanish at the endpoints of $\Gamma$, which will turn out to be true) gives

$$
\int_{\Gamma} e^{i u}\left\{-\left[u\left(u^{2}+\alpha^{2}\right) \phi(u)\right]^{\prime}+\left(u^{2} / 2+\alpha^{2} u+\alpha^{2} / 2\right) \phi(u)\right\} d u=0 .
$$

Thus $\phi$ must satisfy the first order equation

$$
u\left(u^{2}+\alpha^{2}\right) \phi^{\prime}+\left(\frac{5}{2} u^{2}-\alpha^{2} u+\alpha^{2} / 2\right) \phi=0 .
$$

We have

$$
\phi=u^{-1 / 2}(u-i \alpha)^{-1-i \tau}(u+i \alpha)^{-1+i \tau}, \quad \tau=\alpha / 2
$$


A change of variable yields

$$
h(t)=\int_{\Omega} e^{u \alpha \prime \prime} u^{-1 / 2}(1-u)^{-1-i \tau}, \quad \tau=\alpha / 2 .
$$

Our three paths will be $\left(0,1^{+}\right),\left(0,-1^{+}\right),\left(i \infty,-1^{+}, 0^{+}, 1^{+}\right)$, in the standard notation for loop paths. The resulting functions can be identified from the work in [4], and we take as solutions, respectively

$$
\begin{aligned}
& v_{1}=\Phi_{1}\left(\frac{1}{2}, 1-i \tau, \frac{1}{2}-i \tau, i t \alpha\right), \\
& v_{2}=\bar{v}_{1}=\Phi_{1}\left(\frac{1}{2}, 1+i \tau, \frac{1}{2}+i \tau,-1,-i t \alpha\right), \\
& v_{3}=t^{3 / 2} \Phi_{2}\left(1+i \tau, 1-i \tau, \frac{5}{2}, i t \alpha,-i t \alpha\right) .
\end{aligned}
$$

We write

$$
v_{1}=\lambda_{0}+\lambda_{1} \alpha t+\cdots .
$$

The standard series representation for $v_{1}$ (see [5, volume 1 , page 225]) shows $\lambda_{n}$ may be written

$$
\lambda_{n}=\frac{\left(\frac{1}{2}\right)_{n} i^{n}}{\left(\frac{1}{2}-i \tau\right)_{n} n !}{ }_{2} F_{1}\left(\begin{array}{l}
n+\frac{1}{2}, 1-i \tau \\
\frac{1}{2}-i \tau+n
\end{array} \mid-1\right)
$$

or, after an application of Kummer's transformation,

$$
\lambda_{n}=\frac{\left(\frac{1}{2}\right)_{n} i^{n} 2^{i \tau-1}}{\left(\frac{1}{2}-i \tau\right)_{n} n !}{ }^{2} F_{1}\left(\begin{array}{l}
-i \tau, 1-i \tau \\
n+\frac{1}{2}-i \tau
\end{array} \mid \frac{1}{2}\right)
$$

Note, however, that the formulas [5, volume 1 , page $225(2),(21)]$ are incorrect. In the first, $(\beta)_{n}$ should read $(\beta)_{m}$. In the second, $\left(\beta^{\prime}\right)_{m}$ should read $\left(\beta^{\prime}\right)_{n}$.

For $n=0,1, \lambda_{n}$ may be evaluated by formulas due to Mitra [8, page 272].

$$
\begin{aligned}
& \lambda_{0}=2^{i \tau-1} \sqrt{\pi} \Gamma\left(\frac{1}{2}-i \tau\right)\left\{\frac{1}{\Gamma((1-i \tau) / 2)^{2}}+\frac{2 i}{\tau \Gamma(-i \tau / 2)^{2}}\right\} \\
& \lambda_{1}=i 2^{i \tau-1} \sqrt{\pi} \Gamma\left(\frac{1}{2}-i \tau\right)\left\{\frac{1}{\Gamma((1-i \tau) 2)^{2}}-\frac{2 i}{\tau \Gamma(-i \tau / 2)^{2}}\right\}
\end{aligned}
$$

By using elementary properties of the gamma function, it is found that

$$
\left|\operatorname{Im}\left(\lambda_{0} \bar{\lambda}_{1}\right)\right|=1 / 4 \cosh \pi \tau \text {. }
$$

We are now ready to identify the $h$, in terms of the $v_{j}$. We identify $h_{1}$ with $v_{1} / \lambda_{0}$, so $a_{1}=\alpha \lambda_{1} / \lambda_{0}$. Thus

$$
h_{1}=\frac{v_{1}}{\lambda_{0}}, \quad h_{2}=\frac{\left(\bar{\lambda}_{0} v_{1}-\lambda_{0} \bar{v}_{1}\right)}{\alpha\left(\lambda_{1} \bar{\lambda}_{0}-\lambda_{0} \bar{\lambda}_{1}\right)}=-\frac{\operatorname{Im}\left(\bar{\lambda}_{0} v_{1}\right)}{\alpha \operatorname{Im}\left(\lambda_{0} \bar{\lambda}_{1}\right)}
$$

A short computation shows

$$
h_{1}-a_{1} h_{2}=\operatorname{Im}\left(\bar{\lambda}_{1} v_{1}\right) / \operatorname{Im}\left(\lambda_{0} \bar{\lambda}_{1}\right) \text {. }
$$


Letting $t \rightarrow t / \alpha$ and using these in (4.3) gives the relationship

$$
\frac{1}{16 \cosh ^{2} \pi \tau}=\left[\operatorname{Im}\left(\lambda_{1} \Phi_{1}\right)\right]^{2}+\left[\operatorname{Im}\left(\lambda_{0} \Phi_{1}\right)\right]^{2}+\frac{2 \tau t^{3}}{9 \cosh ^{2} \pi \tau} \Phi_{2}^{2},
$$

where $\lambda_{0}, \lambda_{1}$ are as in (4.5), (4.6) and

$$
\begin{aligned}
& \Phi_{1}=\Phi_{1}\left(\frac{1}{2}, 1-i \tau, \frac{1}{2}-i \tau,-1, i t\right), \\
& \Phi_{2}=\Phi_{2}\left(1+i \tau, 1-i \tau, \frac{5}{2}, i t,-i t\right) .
\end{aligned}
$$

Letting $\tau \rightarrow 0$ gives (1.1).

\section{More Horn functions}

This example is involved, but also important because of its application to the theory of nonlinear splines. It results from the simple choice $A=s+\varepsilon$. (The $\varepsilon$ subterfuge is required here because, although the solutions of the equation are analytic (in fact, entire), the vectors $\rho_{j}(\varepsilon)$ are linearly dependent for $\varepsilon=0$.)

The equation is

$$
(s+\varepsilon) \rho^{\prime \prime \prime}-\rho^{\prime \prime}+\alpha^{2}(s+\varepsilon)\left[1+(s+\varepsilon)^{2}\right] \rho^{\prime}-\alpha^{2} \rho=0 .
$$

It is preferable to work in the variable $s$. The scalar equation has a basis of solutions

$$
\begin{aligned}
& \rho_{1}(s+\varepsilon)=(s+\varepsilon)+a_{1}(s+\varepsilon)^{3}+a_{2}(s+\varepsilon)^{5}+\cdots, \\
& \rho_{2}(s+\varepsilon)=(s+\varepsilon)^{3}+b_{1}(s+\varepsilon)^{5}+b_{2}(s+\varepsilon)^{7}+\cdots, \\
& \rho_{3}(s+\varepsilon)=1+c_{1}(s+\varepsilon)^{2}+c_{2}(s+\varepsilon)^{4}+\cdots
\end{aligned}
$$

We have $c_{1}=\alpha^{2} / 2$.

Proceeding as before, we get

$$
\rho(s)=\frac{-\left(1+O\left(\varepsilon^{2}\right)\right)}{6 \varepsilon}\left|\begin{array}{llll}
(0,0,0) & \rho_{1} & \rho_{2} & \rho_{3} \\
(0,0,-1 / \alpha) & \varepsilon & 0 & 1 \\
(0,1,0) & 1 & 0 & -\alpha^{2} \varepsilon \\
(\alpha \varepsilon, 0, \alpha) & 6 a_{1} \varepsilon & 6 \varepsilon & -\alpha^{2}
\end{array}\right| .
$$

Adding $\alpha^{2}$ times the second row to the last, expanding and letting $\varepsilon \rightarrow 0$ gives

$$
\rho(s)=-\left(0,0, \rho_{3} / \alpha\right)-\left(0,\left(a_{1}+\alpha^{2} / 6\right) \rho_{2}-\rho_{1}, 0\right)+\left(\alpha \rho_{2} / 6,0,0\right) \text {, }
$$

so

$$
1 / \alpha^{2}=\rho_{3}^{2} / \alpha^{2}+\left[\left(a_{1}+\alpha^{2} / 6\right) \rho_{2}-\rho_{1}\right]^{2}+\alpha^{2} \rho_{2}^{2} / 36
$$


There remains the problem of identifying $\rho_{f}$. Let

$$
(s+\varepsilon)^{2}=t, \rho(s)=t^{1 / 2} \mathrm{~h}(t) .
$$

The appropriate scalar equation is

$$
8 t h^{\prime \prime \prime}+20 h^{\prime \prime}+2 \alpha^{2}(1+t) h^{\prime}+\alpha^{2} h=0 .
$$

Assume

$$
h=\int_{\Gamma} e^{\prime u} \phi(u) d u
$$

and proceed as in the previous section. $\phi$ is easily determined and, after a change of variable, we have

$$
h=\int_{\Omega} e^{2 i \tau r u} u^{-1 / 2}(1-u)^{-i \tau}(1+u)^{i \tau} d u, \quad \tau=\alpha / 4 .
$$

The solutions we shall use are

$$
\begin{aligned}
& v_{1}=\Phi_{1}\left(\frac{1}{2},-i \tau, \frac{3}{2}-i \tau,-1,2 i \tau t\right), \\
& v_{2}=\bar{v}_{1}=\Phi_{1}\left(\frac{1}{2}, i \tau, \frac{3}{2}+i \tau,-1,-2 i \tau t\right), \\
& v_{3}=t^{-1 / 2} \Phi_{2}\left(i \tau,-i \tau, \frac{1}{2}, 2 i \tau t,-2 i \tau t\right) .
\end{aligned}
$$

Write

$$
v_{1}=\lambda_{0}+\lambda_{1} \tau t+\cdots
$$

Then

$$
\begin{aligned}
\lambda_{0} & ={ }_{2} F_{1}\left(\begin{array}{c}
\frac{1}{2},-i \tau \\
\frac{3}{2}-i \tau
\end{array} \mid-1\right) \\
& =-2^{i \tau+1} \sqrt{\pi} \Gamma\left(\frac{3}{2}-i \tau\right)\left[\frac{1}{\Gamma(-i \tau / 2) \Gamma(1-i \tau / 2)}-\frac{1}{\Gamma((1-i \tau) / 2)^{2}}\right], \\
\lambda_{1} & =\frac{i}{\left(\frac{3}{2}-i \tau\right)}{ }_{2} F_{1}\left(\begin{array}{c}
\frac{3}{2},-i \tau \\
\frac{5}{2}-i \tau
\end{array} \mid-1\right)=\frac{2^{i \tau} i}{\left(\frac{3}{2}-i \tau\right)}{ }_{2} F_{1}\left(\begin{array}{l}
1-i \tau,-i \tau \mid \frac{1}{2} \\
\frac{5}{2}-i \tau
\end{array}\right) .
\end{aligned}
$$

By the use of contiguous relationships for the ${ }_{2} F_{1}$ (see [5, volume 1, page 103 (30)]) we may write

$$
\begin{aligned}
& \lambda_{1}=\frac{8 i 2^{\prime \tau}}{3}\left\{\frac{1}{2}\left(\frac{1}{2}-i \tau\right){ }_{2} F_{1}\left(\begin{array}{l}
1-i \tau,-i \tau \\
\frac{1}{2}-i \tau
\end{array} \mid \frac{1}{2}\right)\right. \\
& +i \tau_{2} F_{1}\left(\begin{array}{l|l}
1-i \tau,-i \tau & \frac{1}{2} \\
\frac{3}{2}-i \tau & 1 \tau
\end{array}\right.
\end{aligned}
$$


so the formulas in [8] may now be applied to get

$$
\lambda_{1}=\frac{2}{3} \sqrt{\pi} \Gamma(3 / 2-i \tau) i\left\{\frac{(1 / 2+2 i \tau)}{\Gamma(-i \tau / 2) \Gamma(1-i \tau / 2)}+\frac{(1 / 2-2 i \tau)}{\Gamma(1 / 2-i \tau / 2)^{2}}\right\} .
$$

For future reference, we need

$$
\left|\operatorname{Im}\left(\lambda_{0} \bar{\lambda}_{1}\right)\right|=\frac{8\left(\tau^{2}+\frac{1}{4}\right)}{3 \cosh \pi \tau} .
$$

We make the identification

$$
a_{1}=\lambda_{1} \tau / \lambda_{0}
$$

and take

$$
\begin{aligned}
& \rho_{1}(s+\varepsilon)=t^{1 / 2} v_{1} / \lambda_{0}, \\
& \rho_{2}(s+\varepsilon)=t^{1 / 2} \frac{\left(\bar{\lambda}_{0} v_{1}-\lambda_{0} \bar{v}_{1}\right)}{\tau\left(\lambda_{1} \bar{\lambda}_{0}-\lambda_{0} \bar{\lambda}_{1}\right)}=\frac{-t^{1 / 2} \operatorname{Im}\left(\bar{\lambda}_{0} v_{1}\right)}{\tau \operatorname{Im}\left(\lambda_{0} \bar{\lambda}_{1}\right)}, \\
& \rho_{3}(s+\varepsilon)=t^{1 / 2} v_{3} .
\end{aligned}
$$

A simple computation gives

$$
a_{1} \rho_{2}-\rho_{1}=-t^{1 / 2} \operatorname{Im}\left(\bar{\lambda}_{1} v_{1}\right) \operatorname{Im}\left(\bar{\lambda}_{1} \lambda_{0}\right) .
$$

Letting $\varepsilon \rightarrow 0$ and replacing $s$ by $s / \sqrt{\tau}$ in (5.5) gives another quadratic relation for $\Phi_{1}, \Phi_{2}$ :

$$
\begin{aligned}
\frac{\left(\tau^{2}+\frac{1}{4}\right)^{2}}{\cosh ^{2} \pi \tau}\left(\Phi_{2}^{2}-1\right) & +\frac{9}{4} s^{2} \tau\left\{\operatorname{Im}\left(\bar{\lambda}_{1} \Phi_{1}\right)+\frac{8 \tau^{2}}{3} \operatorname{Im}\left(\bar{\lambda}_{0} \Phi_{1}\right)\right\}^{2} \\
& +s^{2} \tau \operatorname{Im}\left(\bar{\lambda}_{0} \Phi_{1}\right)^{2}=0,
\end{aligned}
$$

where $\lambda_{0} \lambda_{1}$ are as in (5.7), (5.8) and

$$
\begin{aligned}
& \Phi_{1}=\Phi_{1}\left(\frac{1}{2},-i \tau, \frac{3}{2}-i \tau,-1,2 i s^{2}\right), \\
& \Phi_{2}=\Phi_{2}\left(i \tau,-i \tau, \frac{1}{2}, 2 i s^{2},-2 i s^{2}\right) .
\end{aligned}
$$

If $\tau \rightarrow 0$ the result is trivial. If higher order terms are incorporated, a result for Fresnel integrals is obtained. We can compute a $\tau$ estimate on $\Phi_{2}$ by splitting the defining sum up as follows:

$$
\Phi_{2}=\sum_{n=0}^{\infty} \frac{(-i \tau)_{n}\left(-2 i s^{2}\right)^{n}}{\left(\frac{1}{2}\right)_{n} n !}+\sum_{m=1}^{\infty} \frac{(i \tau)_{m}\left(2 i s^{2}\right)^{n}}{\left(\frac{1}{2}\right)_{m} m !}+\sum_{m, n>0} .
$$

We obtain, after some series manipulations,

$$
\Phi_{2}=1-8 \tau s^{2}{ }_{2} F_{3}\left(\begin{array}{r}
\frac{1}{2}, 1 \\
\frac{3}{2}, \frac{3}{4}, \frac{5}{4}
\end{array} \mid-s^{4}\right)+O\left(\tau^{2}\right) .
$$


Also

$$
\Phi_{1}\left(\frac{1}{2}, 0, \frac{3}{2},-1,2 i s^{2}\right)=\Phi\left(\frac{1}{2}, \frac{3}{2} ; 2 i s^{2}\right), \quad \lambda_{0}=1, \lambda_{1}=2 i / 3,
$$

and, using the formulas [5, volume 1, page 267(29), (30)] we find, in the standard notation for Fresnel integrals,

$$
\operatorname{Re} \Phi_{1}^{2}+\operatorname{Im} \Phi_{1}^{2}=\frac{\pi}{2}\left[C^{2}\left(2 s^{2}\right)+S^{2}\left(2 s^{2}\right)\right] .
$$

Now dividing by $\tau$, letting $\tau \rightarrow 0$ and introducing a change of variable gives

$$
C^{2}(t)+S^{2}(t)=\frac{2 t}{\pi}{ }_{2} F_{3}\left(\begin{array}{c|c}
\frac{1}{2}, 1 \\
\frac{3}{2}, \frac{3}{4}, \frac{5}{4}
\end{array} \mid-\frac{t^{2}}{4}\right), \quad t \geqslant 0 .
$$

This result is not so obscure as it may seem. In fact, it is a direct consequence of the formula [5, volume 1, page 186(5)] which gives

$$
(\operatorname{Re} \Phi)^{2}+(\operatorname{Im} \Phi)^{2}={ }_{2} F_{3}\left(\begin{array}{c}
\alpha, p-\alpha \\
p, \frac{p}{2}, \frac{p+1}{2}
\end{array} \mid-\frac{t^{2}}{4}\right)
$$

where $p$ is such that the ${ }_{2} F_{3}$ is defined, $p$, $\alpha$, are real and

$$
\Phi=\Phi(\alpha, p ; i t) \text {. }
$$

Thus

$$
{ }_{2} F_{3}\left(\begin{array}{c}
\alpha, p-\alpha \\
p, \frac{p}{2}, \frac{p+1}{2}
\end{array} \mid-\frac{t^{2}}{4}\right) \geqslant 0,
$$

an elementary positivity result for $\mathrm{a}_{2} F_{3}$. This should be compared with the positivity statements for ${ }_{1} F_{2}$ 's given in [6], which, however, are much deeper.

\section{Nonlinear splines}

The geometrical aspect of the work in the previous sections has a direct connection with the theory of splines.

In an earlier paper [9], one of us showed how to obtain the intrinsic equations, i.e. the equations specifying $\kappa$ and $\tau$, for a basic spline element $\rho$, from a variational criterion:

$$
\int \kappa^{2} d s=\int\left|\boldsymbol{\rho}^{\prime \prime}\right|^{2} d s=\min
$$

A differential equation was obtained for $\kappa^{2}$,

$$
\left[\left(\kappa^{2}\right)^{\prime}\right]^{2}+\kappa^{2}\left[\left(\kappa^{2}-4 D\right)^{2}-4 A^{2}\right]+4 C^{2}=0
$$


where $A, D, C$ are arbitrary parameters, and for $\tau$,

$$
\kappa^{2} \tau=C \text {. }
$$

Equation (6.2) was reduced to the standard differential equation for the $P$-function of Weierstrass, and it was shown how $\rho(s)$ could be found as a solution of the standard Lamé differential equation.

For planar curves this result simplifies considerably. The torsion vanishes identically and $C$ is zero. The curve $\rho$ may then be written in terms in standard elliptic integrals. If $\kappa^{2} \ll 4 D, \kappa^{2}$ satisfies an approximate equation

$$
\left[\left(\kappa^{2}\right)^{\prime}\right]^{2}-4 \alpha^{2} \kappa^{2}+4 C^{2}=0
$$

whose solution is

$$
\kappa^{2}=\alpha^{2}(s-\sigma)^{2}+C^{2} / \alpha^{2},
$$

where $\sigma$ an integration constant. Again, the matter simplifies considerably for the planar curve, and the result is the Cornu spiral, where $\rho$ has coordinates which can be expressed in terms of the Fresnel integrals.

With $\kappa$ and $\tau$ known from (6.3) and (6.5), $\mathbf{n}$ and $\mathbf{b}$ can be eliminated from the Frenet-Serret formulas to get a third order differential equation for $\mathbf{t}=\boldsymbol{\rho}^{\prime}$ :

$$
\mathbf{t}^{\prime \prime \prime}+\left(\alpha^{2} s^{2}+C^{2} / \alpha^{2}\right) \mathbf{t}^{\prime}+3 \alpha^{2} s \mathbf{t}=\mathbf{0}
$$

or

$$
\boldsymbol{\rho}^{\prime V}+\left(\alpha^{2} s^{2}+C^{2} / \alpha^{2}\right) \boldsymbol{\rho}^{\prime \prime}+3 \alpha^{2} s \boldsymbol{\rho}^{\prime}=\mathbf{0} .
$$

After multiplication by $s$ the left hand side of this equation becomes an exact differential which can be integrated to yield

$$
s \rho " \prime \prime-\rho^{\prime \prime}+s\left(\alpha^{2} s^{2}+C^{2} / \alpha^{2}\right) \rho^{\prime}-\left(C^{2} / \alpha^{2}\right) \rho^{\prime}-\left(-C^{2} / \alpha^{2}\right) \rho_{0}
$$

where the constant of integration has been denoted $\left(-C^{2} / \alpha^{2}\right) \rho_{0}$. If we define a new vector $\eta$ by

then

$$
\eta=\rho-\rho_{0}
$$

$$
s \eta^{\prime \prime \prime}-\eta^{\prime \prime}-\alpha^{2} s\left(s^{2}+C^{2} / \alpha^{2}\right) \eta^{\prime}-\left(C^{2} / \alpha^{2}\right) \eta=\mathbf{0} .
$$

One can, without loss of generality, take $C=\alpha^{2}$ (for, let $s \rightarrow s C / \alpha$ and redefine $\alpha)$. One then obtains the equation of Section 5. The relevant spline element thus lies on a sphere centered at $\rho_{0}$, and its coordinates satisfy essentially the relationship given in (5.9).

\section{Conclusions}

The previous analysis admits to a natural generalization to four dimensions. The condition for the radius vector to touch the hypersphere is the same, but the intrinsic equation is more complicated, containing an additional "hyper" torsion term which measures how the curve twists through four-space. 
Another approach is to require the curve to lie on an ellipsoid of revolution. The final equations will then express the fact that $|\rho-\mathbf{a}|^{2}+|\rho-\mathbf{b}|^{2}=c^{2}$ for some constant vectors $\mathbf{a}$ and $\mathbf{b}$.

\section{Acknowledgment}

The authors acknowledge the helpful suggestions of the referees.

\section{References}

[1] R. Askey, "Jacobi polynomials, I.", SIAM J. Math. Anal. 5 (1974), 119-124.

[2] R. Askey (ed.), Theory and application of special functions (Academic Press, New York, 1975).

[3] L. P. Eisenhart, An introduction to differential geometry (Princeton University Press, 1947).

[4] A. Erdélyi, "Some confluent hypergeometric functions of two variables", Proc. Roy. Soc. Edinburgh 60 (1939-1940), 341-361.

[5] A. Erdèlyi et al., Higher transcendental functions, 3 volumes (McGraw-Hill, New York, 1953).

[6] J. L. Fields and M. E. Ismail, "On the positivity of some ${ }_{1} F_{2}$ 's", SIAM J. Math. Anal. 6 (1975), 551-559.

[7] E. L. Ince, Ordinary differential equations (Dover, New York, 1956).

[8] Y. L. Luke, Mathematical functions and their approximations (Academic Press, New York, 1975).

[9] E. Mehlum, "Nonlinear splines", in Computer aided geometric design (eds. R. E. Barnhill and R. F. Riesenfield), (Academic Press, New York, 1974).

[10] E. Mehlum, "Appell and the apple (Nonlinear splines in space)", Report, Sentralinstitutt for industriell forskning, Oslo. (This report is obtained by writing to SI, Forskningsveien 1, Oslo 3, Norway.)

[11] D. J. Struik, Lectures on classical differential geometry (Addison-Wesley, Reading, Massachusetts, 1950).

[12] G. N. Watson, A treatise on the theory of Bessel functions, 2nd ed. (Cambridge University Press, 1962). 